\title{
Fog computing for assisting and tracking elder patients with neurodegenerative diseases
}

\author{
Iván García-Magariño ${ }^{1,2}$ • José Varela-Aldas ${ }^{3}$. Guillermo Palacios-Navarro ${ }^{4}$. Jaime Lloret $^{5}$
}

Received: 13 July 2018 / Accepted: 17 February 2019

C) Springer Science+Business Media, LLC, part of Springer Nature 2019

\begin{abstract}
U.S. hospitals transmit and manage great amounts of information with the avenue of Internet of things. This work departs from a real need detected by healthcare centers and hospitals in U.S., Spain and Ecuador. This work focuses on the application of fog computing for obtaining an app rich in visual content that will not overload U.S. hospital infrastructures even if it was used massively. The simulation results showed that the proposed fog-based approach could support a regular use (one day out of three on average) by $1 \%$ of patients of one of the most common neurodegenerative diseases in 14 states in U.S (i.e. 36,400 patients in total) with only a traffic of $528 \mathrm{~KB}$ per day on average when using one hospital per state.
\end{abstract}

Keywords Fog computing $\cdot$ Neurodegenerative disorder $\cdot$ Virtual reality $\cdot$ Mobile application $\cdot$ Agent-based simulation

\section{Introduction}

Hospitals are integrating the use of Internet of Things (IoT) in medical devices for life-critical health monitoring, increasing the demand for greater bandwidth for communications. Emergent solutions in medical IoT are facing challenges such as the information sharing and collaboration among heterogeneous sensor-enabled medical devices and battery lifecycle of the corresponding wearable and portable devices [1]. Fog computing can reduce the bandwidth of communications by augmenting the local processing and only transmitting the essential amount of information required by the hospital and healthcare providers [2].

Clinical trials involving the transmission of images are one of the overloading activities on the bandwidth of healthcare networks [3]. More concretely, the assessment of

This article is part of the Topical Collection: Special issue on Fog Computing for Healthcare

Guest Editors: Han-Chieh Chao, Sana Ullah, Christos Verikoukis, and Ki-Il Kim

Jaime Lloret

jlloret@dcom.upv.es

Extended author information available on the last page of the article. patients with neurodegenerative diseases normally requires using images, so patients perceive visual stimuli.

Continuous remote health monitoring is gaining relevance in the late years for allowing caregivers to keep track of some health indicators. For example, a smart system is now able to monitor children with chronic illness by means of wearable sensors and a smartphone, and this alerts the caregivers when some indicators such as heart rate or body temperature surpass certain thresholds [4]. In addition, there is an architecture for remote monitoring in e-Health systems [5]. This architecture relies on using 5G networks for using a proper bandwidth. It also uses big data analytics for alerting of anomalous situations by analyzing the information collected by wearable sensors. In addition, the field of mobile health applications with efficient management of data transmission is growing with a high impact in the society. For instance, an Android-based self-managed mobile application can assist users in collecting and monitoring health indicators for warning care services when appropriate [6]. This app provides self-care for people with chronic illnesses, and constitutes an example of how hospitals can facilitate efficient data communications for telecare.

Fog computing allows e-Health systems to reduce the necessary bandwidth by managing the information more efficiently and in a more distributed way [7]. This is achieved by analyzing most of the data locally and only 
sending the summarized relevant information to a cloud [8]. The existing applications of fog computing for e-Health have different purposes. For example, fog computing has been applied for long-term monitoring of Electrocardiogram (ECG) signals [9], but it has also been applied for monitoring patients with mild dementia in smart home environments for ambient-assisted living [10]. However, to the best of the authors' knowledge, fog computing has not been applied yet for assisting and tracking the evolution of elder people through with neurodegenerative disease through an application of virtual reality (VR).

In this context, we have developed a mobile application for the early detection and tracking of some neurodegenerative disorders with VR. This app relies on the fundamentals of fog computing, in which the images and 3D scenes are stored locally, and the user's replies and actions are processed locally as well. Each app instance sends the data to the local hospital following the principles of fog computing. In this approach, hospitals only interchange patients' data when necessary, for example when a patient changes his or her residence from one city to another or in case of emergencies.

This article is organized as it follows. The next section reviews the most relevant related work, highlighting the main literature gap covered by the proposed fog-based approach. Section 3 introduces the materials and methods of the proposed fog-based approach, including the novel app for elder people with neurodegenerative disorders with a fog-computing approach and the evaluation method of the experiments. Section 4 presents the main results of the experiments discussing the most relevant aspects. Finally, Section 5 mentions the conclusions and depicts some future research lines.

\section{Related work}

The emerging technologies in machine-to-machine (M2M) communications have allowed to interconnect a wide range of wireless devices for implementing $\mathrm{m}$-health applications [11]. Wireless body area networks are the most common ones in the patient's side, but M2M technologies also support applications that can benefit healthcare providers. In the context of ambient assisted living (AAL), Kartsakli et al. [12] proposed a communication framework that kept data exchange and storage at a local data plane, but moved the coordination to the cloud. Their framework was mainly designed to support healthcare applications, and showed performance gains. This system is related with some principles of fog computing since most of the processing and exchange was performed locally. However, their approach was more focused on AAL rather than tracking the development of people with neurodegenerative disorders through data collection from technologicallysupported cognitive assessment.

Several works have specifically applied fog computing for health systems. For example, the prototype Smart eHealth Gateway called UT-GATE [7] uses a geo-distributed intermediary layer for managing the information of sensor nodes and storing these in the cloud. They evaluated their approach with a health monitoring system with an assessment platform based on IoT early-warning scores. In addition, Mahmoud et al. [13] presented a fog-computing strategy for managing IoT information from healthcare systems before uploading to the cloud. It places some tasks of applications in fog devices with an energy-aware allocation strategy. Their experiments with the iFogSim simulator showed the energy-consumption reduction of their approach over the cloud-only strategy and the fogdefault one. Furthermore, Wu et al. [9] applied fog computing for long-term monitoring of ECG signals with a t-shirt as the wearable carrier of the corresponding sensors. They evaluated tactile comfort, signal to noise ratio and thermal conductivity, obtaining promising results. Fog-computing has also been applied in the context of ambient assisted-living [10]. In particular, eWALL fits the regulations and procedures requirements for patients with mild dementia. It used a computational-distributed approach following the fog computing principles. The home environment processed the sensed information for not overloading the communications.

Agent-based simulators (ABSs) have simulated communications in different network types for assessing these. For instance, an ABS simulated the communications of a mobile ad hoc network (MANET) in battlefield for mission-critical military operations [14]. This ABS considered several perturbation factors such as noises and enemy attacks and their repercussions in the wireless communication topology of the MANET. They showed how the soldiers' collective movements positively affected to the capacity of the communication channels. Another ABS simulated the communications for the coordination of electricity distribution in smart grids [15]. The simulated outcomes showed an adequate demand response. The ABS assessed an approach about control capabilities in smart grids when there were communication constraints. Therefore, in general ABSs have proved to be useful for assessing different communication approaches.

With the advent of mobile technologies, several VR applications have been designed to assist in the diagnosis and treatment of elder people with neurodegenerative disorders. Ouellet et al. [16] recently published a study that examined the ability of a VR supermarket task to distinguish between younger and older adults, as well as individuals with and without subjective cognitive complaints. Montenegro and Argyriou [17] recently presented a game-based 
Peer-to-Peer Netw. Appl.

application for diagnosing neurodegenerative disease. In this application, individuals complete a task similar to the Turing test in a virtual environment, and the VR application distinguished between healthy people and individuals with the neurodegenerative disease. Doniger et al. [18] recently proposed a randomized control trial for a cognitive training VR program for cognitively normal adults at high risk for a neurodegenerative disease based on family history. Their application was designed to train the capacity of remembering and executive functions (e.g., planning) by having individuals engage in a VR shopping experience in a supermarket. Participants in the proposed VR study will look for items on a grocery list and would put the items in the grocery cart. The authors plan to assess whether this app will improve cognition and cerebral blood flow in participants. In this proposed clinical trial, VR is believed to replicate the complexity of daily activities well and help train in the skills normally affected in neurodegenerative disorders.

Nevertheless, none of the aforementioned works applied fog computing for assisting and tracking elder people with neurodegenerative disorders for not overloading communications.

\section{Materials and methods}

The main material used in this research is the app for assisting and tracking elder people with neurodegenerative disorders with a fog-computing approach, which is introduced in Section 3.1. In addition, Section 3.2 describes the method followed for evaluating the proposed fog-based approach with agent-based simulation.

\subsection{App with fog computing for elder patients with neurodegenerative disorders}

Figure 1 shows the fog computing approach designed in this app. All the images and 3D scenes are stored locally in each mobile device. In this way, the transmission of images does not overload the network. The actions of patients in this app are used to evaluate some of their features. This assessment is done locally by counting the correct answers and applying the corresponding weight factors. This also saves communication bandwidth. The proposed fog-based approach only sends the final evaluation results represented as a few numbers, which is a low amount of information. Therefore, most of the processing is performed locally.

Moreover, following the principles fog computing approach, each patient is associated with their affiliated hospital. The app only sends data to the server of this hospital. In this way, the possibility that the data server of each hospital becomes a bottleneck is reduced, as the gathering of data is distributed between the different hospitals. The doctors of a hospital can directly access to the assessment results of the patients of their hospital. In the occasional cases in which a doctor needs to access to the assessment data of a patient from another hospital, then the patient's hospital sends this information to the current hospital. This should only occur in emergencies or changes of residences. In the latter case, the history data would be
Fig. 1 Architecture of the proposed fog-based approach in the presented app

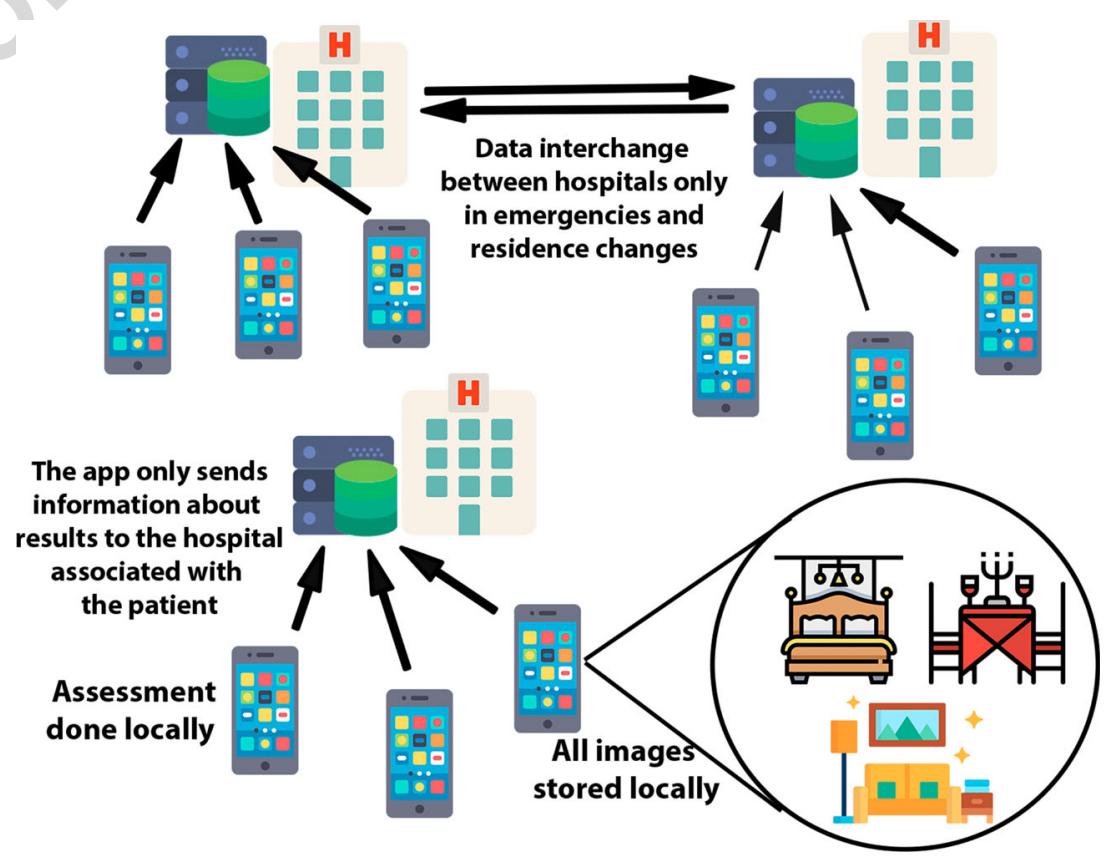


moved, and then the patient's app would be associated to their new hospital.

We developed this app in an international collaboration between University of Zaragoza and Technological University Indoamérica Guayaquil and Simón Bolivar addressing a real need detected U.S., Spain and Ecuador.

Figure 2 shows the block diagram of the functioning of the presented app. Besides the common modeling elements for actions and flow bifurcations, this diagram uses a specific notation for referring to the sending of messages. The right-bottom area of the diagram includes a legend indicating the meaning of all the used notations. It uses several scenarios for assisting elder patients with neurodegenerative diseases. For each scenario, it asks several questions or ask them to perform certain actions. Then, it asks the user to perform a task of the whole scenario. After testing all the scenarios, the app asks the user to perform a final task about some of the previous scenarios.
All the results are assessed locally, and only the final results are sent to the associated hospital.

The proposed app has been developed with FAMAP (a Framework for developing M-health Apps) [19], which helped us in the generic definition of test questions. The module of questionnaires was extended to allow including images in the test answer options. This app was developed with C\# programming language and the Unity $3 \mathrm{D}$ environment.

\subsection{Evaluation method using agent-based simulation}

In order to test the performance of the proposed fog-based approach, we simulated the estimated data traffic of the app when it is widespread among some states of U.S. In order to conduct a simulation with accurate figures, we extracted the data from a official report in 2017 about one of the most
Fig. 2 Block diagram of the proposed fog-based approach

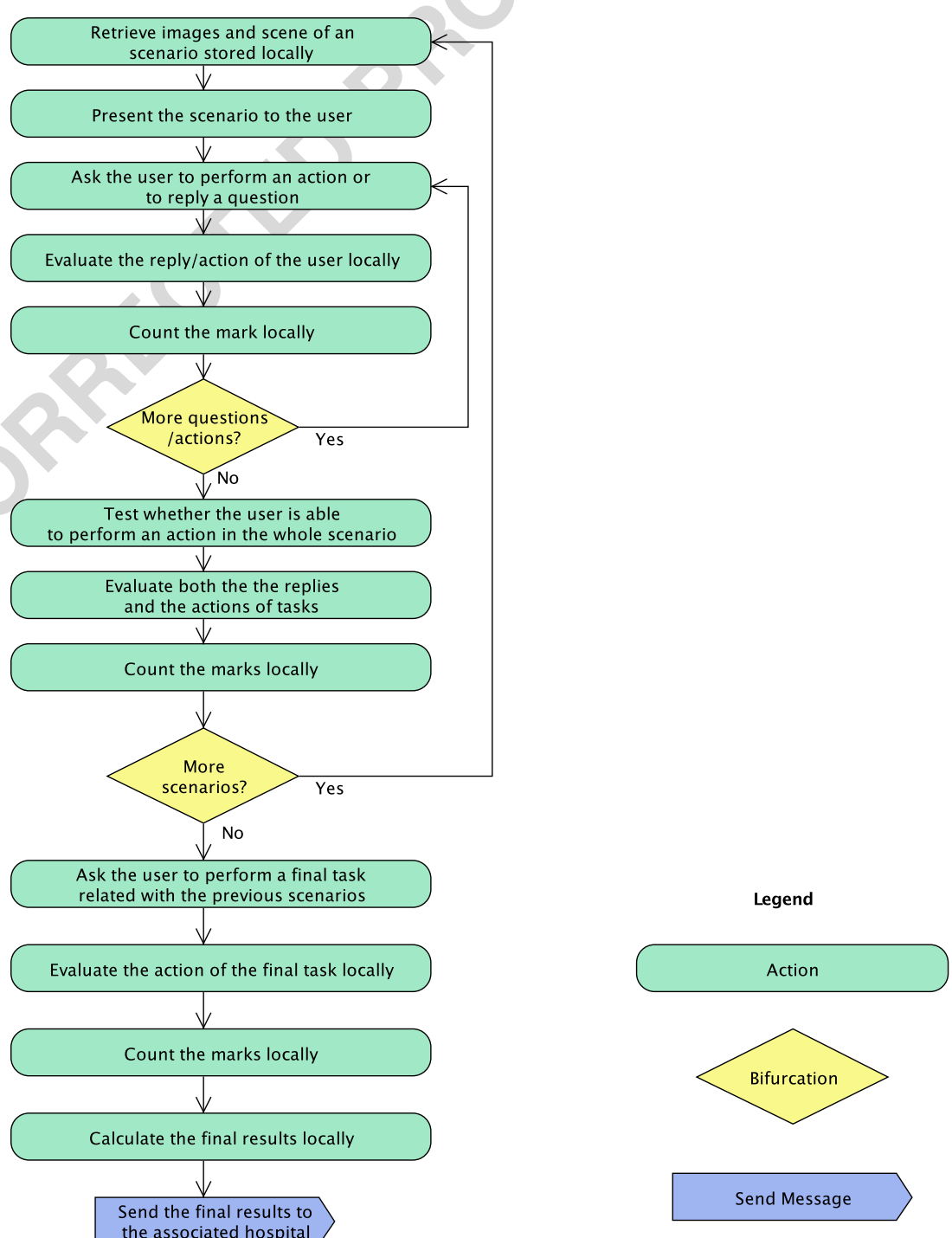


common neurodegenerative diseases [20]. In particular, we selected all the states that had 120,000 patients of this disease or a greater number according to this report. In particular, these states with the number of patients expressed in thousands were Arizona (130), California (630), Florida (520), Georgia (140), Illinois (220), Massachusetts (120), Michigan (180), New Jersey (170), New York (390), North Carolina (160), Ohio (210), Pennsylvania (270), Texas (360) and Virginia (140).

To compare the proposed fog-based approach with alternatives, we simulated the traffic load with the same test as in the proposed app, but using other different approaches. One of these approaches used a web service in which the user just needed to access a web address with a browser. This alternative will be referred as "web service" from this point forward. The second approach was an app that stored locally the images and performed the processing, but it sent all the data results to the same central server of a particular hospital. This second alternative is referred as "alternative app" from now on.

In order to perform the simulation, we have used an ABS about the transmission of information for the different tested options. For this purpose, we have used the approach presented in TABSAOND (a technique for developing ABS apps and online tools with nondeterministic decisions) [21] for simulating the nondeterministic decisions. We selected TABSAOND because it provides a wider range of options for selecting non-deterministic decisions in comparison to other alternative ABS environments such as NetLogo and Repast Simphony. The non-deterministic decisions were appropriate for simulating people deciding when to use use the app. More concretely, we used a normal distribution for simulating the distribution of people connecting to the app, which is one of the options recommended by TABSAOND. We applied a standard deviation (SD) of 0.2 over the normal distribution of probability from 0 to 1 . We used a mean of 0.5 in the interval from $[0,1]$. In this way random numbers homogeneously distributed in this interval were converted in values following a normal distribution, which is usually much more similar to the reality.

In order to improve the performance of the ABS, each agent simulated a group of people, as commonly done in the ABS literature (see an example in [22] in tourism domain where each agent could represent a family or a group of friends) and as recommended by PEABS (a process for developing efficient agent-based simulators) [23]. We selected PEABS instead of other general-purpose development processes such as the Scrum agile process or the iterative Rational Unified Process (RUP), because PEABS fastened the development of the ABS since it was supported with a framework for the straightforward definition of agents. We chose PEABS instead of other agent-development processes such as the ones commonly followed in Ingenias and Prometheus methodologies, because PEABS allowed us to obtain a more efficient software product (i.e., the ABS) in terms of both response time and feasible amounts of agents.

In the ABS, each agent simulated the number of tests done by the patients of each state in each day, with the following formula:

$t=\left(\frac{1}{\sqrt{2 \pi \sigma^{2}}} e^{-\frac{(x-\mu)^{2}}{2 \sigma^{2}}}\right) \cdot f \cdot a \cdot u$

where $\sigma$ and $\mu$ are respectively the aforementioned SD and mean associated with the normal distribution of probabilities, $x$ is a random number in the $[0,1]$ interval generated in each day a state, $f$ is the frequency of tests per day (e.g. 0.333 if considering one test every three days in average), $a$ is the number of patients of this disease in the corresponding state, and $u$ is the ratio of the simulated regular users between the existing patients of this disease.

A month of 30 days was simulated considering the 14 aforementioned U.S. states. The simulations presented both the number of requests to the server (also referred as number of connections) and the data transmitted. We calculated the number of requests per test and the data transmitted for the proposed fog-based approach and each of the alternatives. The total data quantities were calculated by multiplying the number of requests by the data transmitted per request.

In this simulation evaluation, we considered that $1.0 \%$ of the patients of this disease of the 14 selected states were regular users of the app. In addition, we simulated that the frequency of users was one out of three days in average. In addition, the amount of data transferred of the proposed fogbased approach were $0.60 \mathrm{~KB}$ considering the assessment results, the identifier of the user, and the encryption to preserve the privacy of the user. This amount also takes into account the necessary information about the network packages such as for example the target IP address. We used the same amount for the alternative app. In order to simulate the data transmitted in the web service, we measured the information transferred considering the sum of the sizes of all the images that were necessary to transfer in each test.

We also calculated the improvement ratios about both number of connections and data transferred of the proposed fog-based approach over the two alternative approaches. We also represented the graphs of the simulation. Firstly, we compared the proposed fog-based approach with the web service, and secondly with the alternative app. The next section presents the results of these comparisons.

\section{Results and discussions}

The results of the simulations provide an estimation of the utility of the proposed fog-based approach in 
terms of integrating the tracking of elder patients with neurodegenerative disorders in hospital servers without overloading the hospital communications in comparison to other alternatives. All the experiments with the proposed fog-based approach and the two alternatives have been executed with the same conditions and input parameters, which were mentioned in Section 3.2.

Figure 3 shows the simulation of the information transferred in the proposed fog-based approach for a month. This figure shows a boxplot that indicates the average, minimum, maximum and the division in quartiles for each U.S. state. In this case, we assumed that each of the 14 states had a hospital with a server that collected the information of the corresponding state. Figure 4 presents the simulation results of the the proposed fog-based approach about the connections to the hospital server in each state with a boxplot. The average data amount that each server needed to transfer was only $528 \mathrm{~KB}$ per day. The most overloaded day in the worst state was $2,472 \mathrm{~KB}$, which can be considered still a very low amount. The average amount of connections was 825 connections per day, and in the worst day of the most overloaded server was 4184 connections. The amounts of transmitted data were related with the population of each state. For example, California and Florida were respectively the first and second with the most transmitted data and they were also the ones with first and second highest populations. Massachusetts and Arizona were the states with the first and second lowest populations, and also were two of the ones with the least transmitted data. The high differences of transmitted data could be possibly reduced by supporting the data collection by more than one hospital in the states with high populations.

In the comparison, the first alternative was to provide the test as a web service. In this case, the result of summing all the image sizes of the test was 23.6 MB. This information needed to be transferred in every test. In addition, the web service connected to the server in every screen the user went through. More concretely, it needed 46 connections per test. In order to make a fairer comparison, we also compared the proposed fog-based approach with an alternative app, which was similar to the proposed one but without using fog computing. In particular, it also managed the images and scenes locally as well as the assessment of the scores. However, this alternative app made all the requests over the same central server with the corresponding database.

Figure 5 compares the amount of data transmitted between the patients and the server between the proposed fog-based approach and the other two alternatives. In each day of this chart, we considered the server with the highest traffic for the proposed fog-based approach. This graph uses a logarithmic scale to properly show large differences. In the web service, the average of data transmitted was $170.8 \mathrm{~GB}$ per day. The amount of information was more than 300,000 times than in the proposed fog-based approach. In the worst day, the server was requested to transfer $238.4 \mathrm{~GB} / \mathrm{day}$, an enormous amount that could provoke the denial of service of most test requests and slow the service down, as well as other requests in the hospital. In the alternative app, the average transmitted data was $7.06 \mathrm{MB} /$ day, and the ratio was 13.80 times more than in the proposed approach considering
Fig. 3 Simulation of data transmitted with the proposed fog-based approach

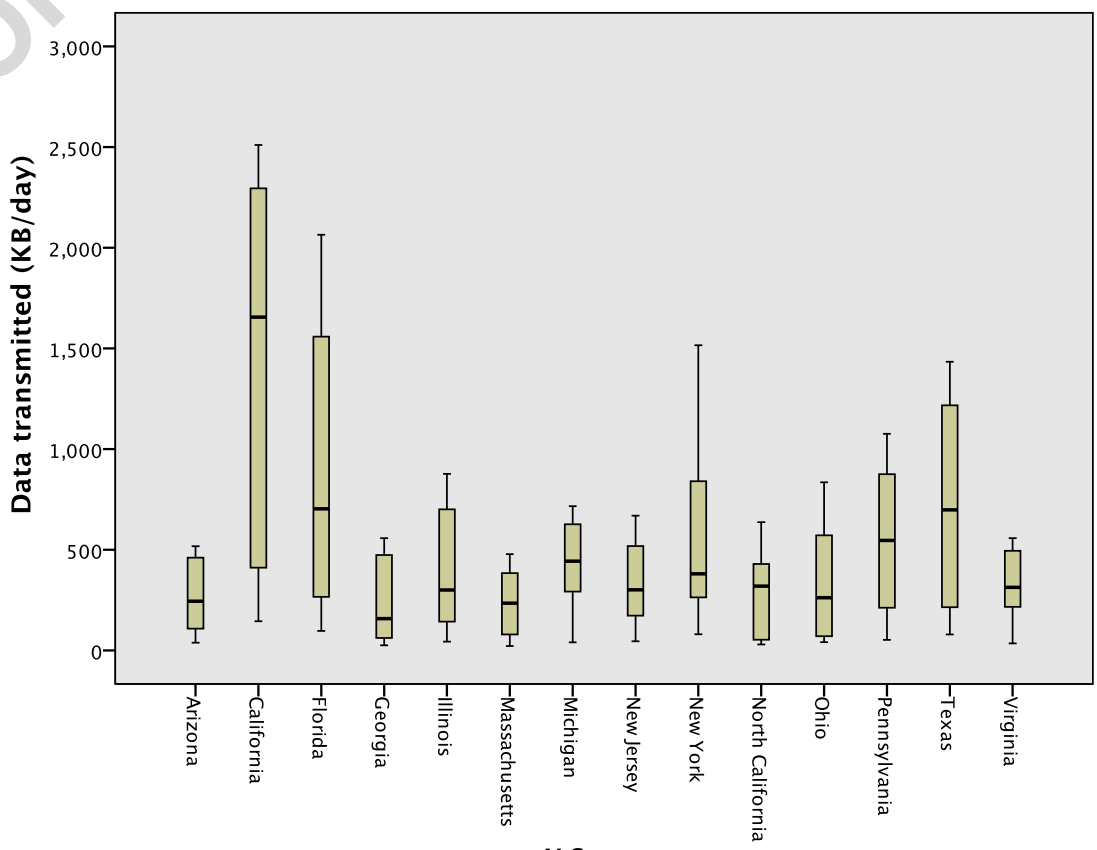

U.S. state 
Peer-to-Peer Netw. Appl.

Fig. 4 Simulation of the number of connections with the proposed fog-based approach

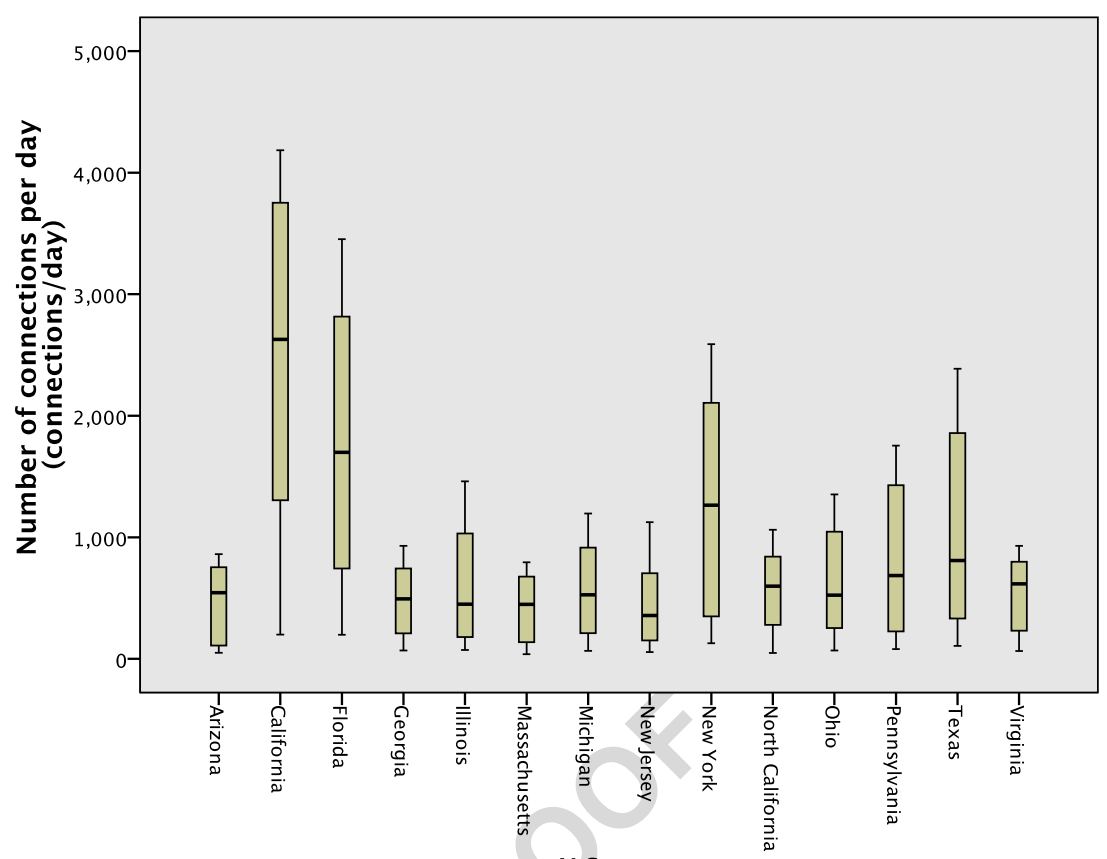

U.S. state

the average of the servers. This value is approximately the number of servers used in this simulation of the fog computing. Thus, one can infer that the traffic load is probably reduced approximately by a ratio about the number of servers used for the proposed fog-computing approach.

In addition, Fig. 6 compares the number of connections between the proposed fog-based approach, the web service and the alternative app. In the proposed fog-based approach, we used the maximum number of connections from all the servers. The web service needed 557,635 connections per day, which was more than 600 times more connections than the proposed fog-based approach. In average, the alternative app performed 12,227 connections per day, and it needed a maximum of 15,789 connections in the worst day. The proposed fog-based approach reduced 14.82 times the average connections of all servers. This number was also similar to the number of servers used in the proposed fog-based approach.

Fig. 5 Comparison of the data transmitted between the proposed fog-based approach, the alternative app and the web service

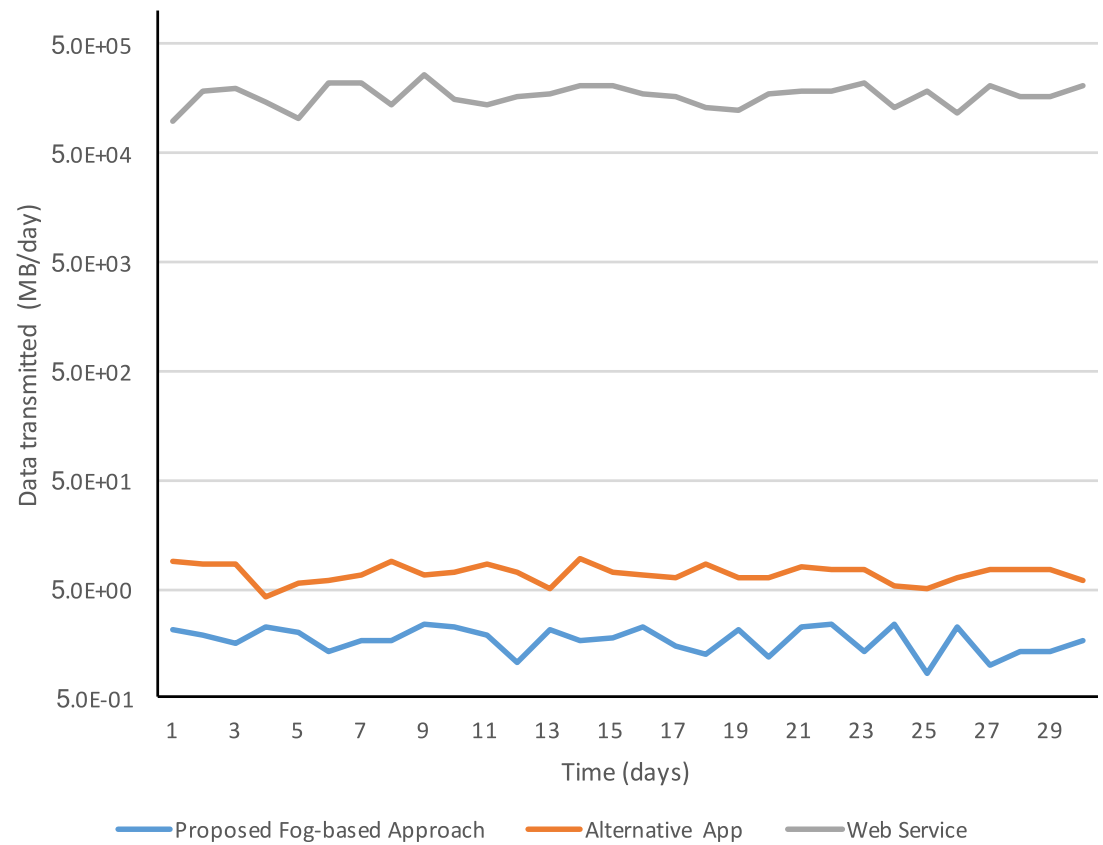


Fig. 6 Comparison of the number of connections per day between the proposed fog-based approach, the alternative app and the web service

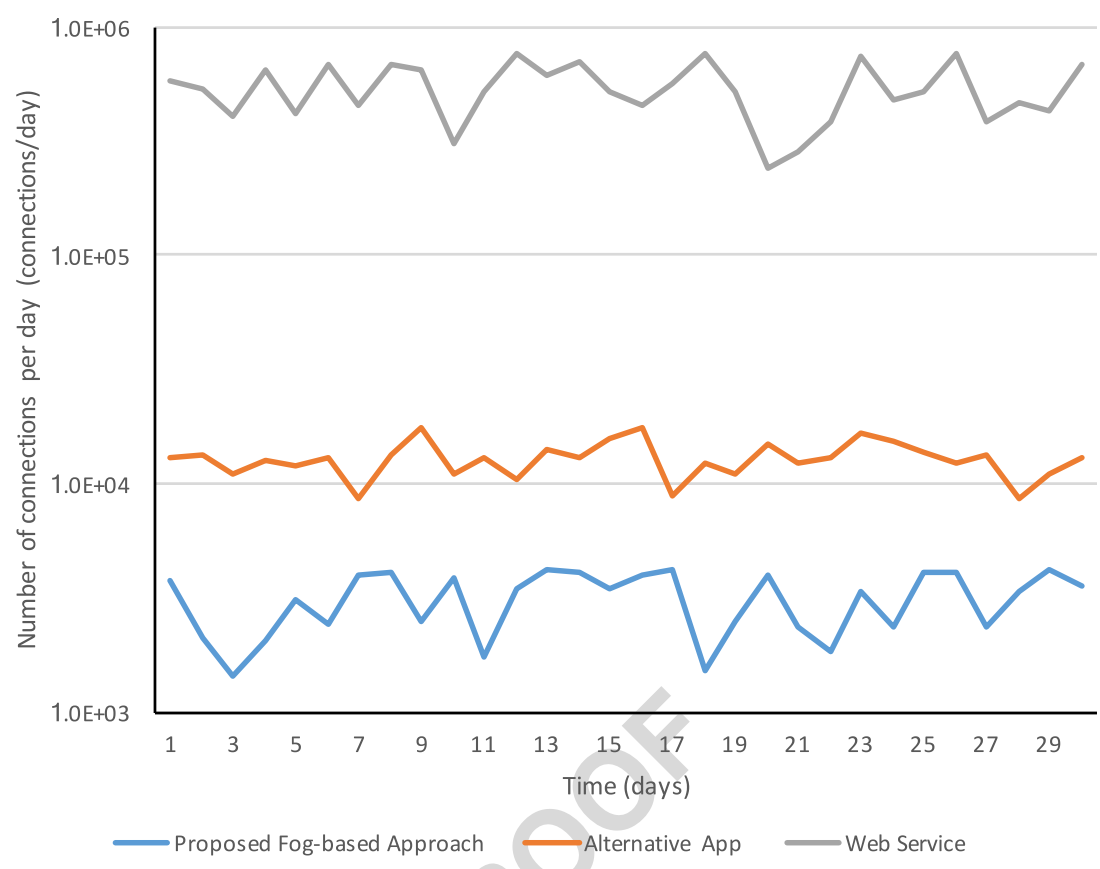

On the whole, one can observe that the proposed fogbased approach could provide an app that potentially assist and track patients with neurodegenerative disorders in the U.S. and barely interfere with the communications of hospitals by assigning the collection of data to different hospitals. The app we developed locally stores all the visual components, and also manages locally the measurement and scoring of the test, only sending the final scores that summarize all the activity of the user. Therefore, this work aligns with the principles of fog computing [8], where the storage of information and processing is mostly performed locally. It also shares the summarized information through a distributed storage system using the servers of different hospitals. In the light of the obtained results, this work recommends to integrate fog computing approach into mobile application tests for patients with neurodegenerative disorders that are rich in visual information.

It is worth mentioning that a limitation of the proposed fog-based approach is that users need to download and install the app with all its visual content. However, this communication transmission would be managed directly by the Google servers through the Google Play store, without overloading the traffic load of hospital servers.

\section{Conclusions and future work}

This work has presented a novel application for potentially assisting and tracking people with neurodegenerative diseases. This paper focuses on the application of fog computing for alleviating the use of bandwidth in hospital servers in case patients massively use the app. According to the simulated results, this app could support the assessment of patients with one of the most common neurodegenerative disease in the 14 states by only using one hospital server per state, and assuming that $1 \%$ of the patients used it regularly.

This work is planned to be extended by validating the measurement of certain features of patients with neurodegenerative diseases. We plan to conduct a pilot normative study with healthy individuals and, if appropriate based on results, a study of patients with a specific neurodegenerative disease. We aim to determine whether the measurements of our app properly correlates with validated scales. In the long-term, we may design a fogcomputing approach for processing brain maps and sharing some summarized relevant information, such as the color histograms for certain regions of the brain.

Acknowledgements This work was performed during the research stay of the first author in the Massachusetts General Hospital and Harvard University, funded by "Dpto. de Innovación, Investigación y Universidad del Gobierno de Aragón" through the program "FEDER Aragón 2014-2020 Construyendo Europa desde Aragón" (Ref: T49_17R). This work also acknowledges the research project "Construcción de un framework para agilizar el desarrollo de aplicaciones móviles en el ámbito de la salud" funded by University of Zaragoza and Foundation Ibercaja with grant reference JIUZ2017-TEC-03. We also acknowledge support from "Universidad de Zaragoza", "Fundación Bancaria Ibercaja" and "Fundación CAI" in the "Programa Ibercaja-CAI de Estancias de Investigación" with reference IT1/18.

Publisher's note Springer Nature remains neutral with regard to jurisdictional claims in published maps and institutional affiliations. 
Peer-to-Peer Netw. Appl.

\section{References}

1. Sodhro AH, Pirbhulal S, Sangaiah AK (2018) Convergence of IoT and product lifecycle management in medical health care. Futur Gener Comput Syst 86:380-391

2. Farahani B, Firouzi F, Chang V, Badaroglu M, Constant N, Mankodiya K (2018) Towards fog-driven IoT eHealth: Promises and challenges of IoT in medicine and healthcare. Futur Gener Comput Syst 78:659-676

3. Collotta M, Pau G, Costa DG (2018) A fuzzy-based approach for energy-efficient Wi-Fi communications in dense wireless multimedia sensor networks. Comput Netw 134:127-139

4. Sendra S, Parra L, Lloret J, Tomás J (2018) Smart system for children's chronic illness monitoring. Inf Fusion 40:76-86

5. Lloret J, Parra L, Taha M, Tomás J (2017) An architecture and protocol for smart continuous eHealth monitoring using $5 \mathrm{G}$. Comput Netw 129:340-351

6. Kao H-Y, Wei C-W, Yu M-C, Liang T-Y, Wu W-H, Wu YJ (2018) Integrating a mobile health applications for self-management to enhance telecare system. Telematics Inform 35(4):815-825

7. Rahmani AM, Gia TN, Negash B, Anzanpour A, Azimi I, Jiang M, Liljeberg P (2018) Exploiting smart e-health gateways at the edge of healthcare Internet-of-things: a fog computing approach. Futur Gener Comput Syst 78:641-658

8. Hu P, Dhelim S, Ning H, Qiu T (2017) Survey on fog computing: architecture, key technologies, applications and open issues. J Netw Comput Appl 98:27-42

9. Wu W, Pirbhulal S, Sangaiah AK, Mukhopadhyay SC, Li G (2018) Optimization of signal quality over comfortability of textile electrodes for ECG monitoring in fog computing based medical applications. Futur Gener Comput Syst 86:515-526

10. Fratu O, Pena C, Craciunescu R, Halunga S (2015) Fog computing system for monitoring Mild Dementia and COPD patientsRomanian case study. In: 2015 12th international conference on telecommunication in modern satellite, cable and broadcasting services (TELSIKS), pp 123-128. Nis, Serbia: IEEE

11. Kartsakli E, Lalos AS, Antonopoulos A, Tennina S, Di Renzo M, Alonso L, Verikoukis C (2014) A survey on M2M systems for mHealth: a wireless communications perspective. Sensors 14(10):18009-18052

12. Kartsakli E, Antonopoulos A, Lalos AS, Tennina S, Di Renzo M, Alonso L, Verikoukis CV (2015) Reliable MAC design for ambient assisted living: moving the coordination to the cloud. IEEE Commun Mag 53(1):78-86
13. Mahmoud MME, Rodrigues JJPC, Saleem K, Al-Muhtadi J, Kumar N, Korotaev V (2018) Towards energy-aware fog-enabled cloud of things for healthcare. Comput Electr Eng 67:5869

14. Regragui Y, Moussa N (2016) Agent-based system simulation of wireless battlefield networks. Comput Electr Eng 56:313333

15. Kilkki O, Kangasrääsiö A, Nikkilä R, Alahäivälä A, Seilonen I (2014) Agent-based modeling and simulation of a smart grid: a case study of communication effects on frequency control. Eng Appl Artif Intell 33:91-98

16. Ouellet E, Boller B, Corriveau-Lecavalier N, Cloutier S, Belleville S (2018) The virtual shop: a new immersive virtual reality environment and scenario for the assessment of everyday memory. J Neurosci Methods 303:126-135

17. Montenegro JMF, Argyriou V (2017) Cognitive evaluation for the diagnosis of Alzheimer's disease based on turing test and virtual environments. Physiol Behav 173:42-51

18. Doniger GM, Beeri MS, Bahar-Fuchs A, Gottlieb A, Tkachov A, Kenan H, Livny A, Bahat Y, Sharon H, Ben-Gal O et al (2018) Virtual reality-based cognitive-motor training for middle-aged adults at high Alzheimer's disease risk: a randomized controlled trial. Alzheimer's \& Dementia: Translational Research \& Clinical Interventions 4:118-129

19. García-Magariño I, Bedia MG, Palacios-Navarro G (2018) FAMAP: a framework for developing m-Health Apps. In: World conference on information systems and technologies, vol 745 of advances in intelligent systems and computing, pp 850-859. Springer

20. Alzheimer's Association et al (2017) 2017 Alzheimer's disease facts and figures. Alzheimers Dement 13(4):325373

21. García-Magariño I, Palacios-Navarro G, Lacuesta R (2017) TABSAOND: a technique for developing agent-based simulation apps and online tools with nondeterministic decisions. Simul Model Pract Theory 77:84-107

22. García-Magariño I (2015) ABSTUR: an agent-based simulator for tourist urban routes. Expert systems with applications 42(12):5287-5302

23. García-Magariño I, Gómez-Rodríguez A, González-Moreno JC, Palacios-Navarro G (2015) PEABS: a process for developing efficient agent-based simulators. Eng Appl Artif Intell 46:104112 